\title{
BEM-ESTAR SUBJETIVO, FUNCIONALIDADE E APOIO SOCIAL EM IDOSOS DA COMUNIDADE
}

\author{
Fabiana Caetano Martins Silva e Dutra ${ }^{1}$ \\ Helena Rita Oliveira Silva²
}

resumo

O envelhecimento é um processo que tem como característica o aumento do nível de incapacidade funcional e diminuição da qualidade de vida. Este processo pode ser influenciado pela rede de suporte social e pelo bem-estar do idoso. $\bigcirc$ objetivo deste estudo foi avaliar o impacto do apoio social na funcionalidade e no bem-estar subjetivo de idosos na comunidade. Trata-se de um estudo quantitativo, observacional, transversal, realizado com 39 idosos cadastrados em equipe de estratégia de saúde da família e participantes de associações de bairro, em Uberaba/MG. Avaliou-se o suporte social pelo domínio de Interação Social do Perfil de Saúde de Nottingham, e a funcionalidade foi avaliada utilizando o BOMFAQ e Bem-Estar

\footnotetext{
1 Terapeuta Ocupacional. Doutora em Ciências da Reabilitação. Professora Adjunta da Universidade Federal do Triângulo Mineiro, vinculada ao Departamento de Terapia Ocupacional. Líder do Núcleo de Estudos e Pesquisas sobre Trabalho, Participação Social e Saúde. E-mail: fabiana@to.uftm.edu.br 2 Terapeuta Ocupacional pela Universidade Federal do Triângulo Mineiro. Pesquisadora do Núcleo de Estudos e Pesquisas em Trabalho, Participação Social e Saúde (NETRAS). E-mail: helenaoliveirato@ gmail.com
} 
pela Escala de Bem-Estar Subjetivo. A amostra caracterizou-se essencialmente por mulheres (74,4\%), com idade variando entre 60 e 82 anos, vivendo casadas ou com companheiro, com baixa escolaridade e renda média mensal de 1,7 salários mínimos. A análise bivariada mostrou correlação significativa entre interação e apoio social e capacidade funcional, bem como correlação significativa entre morar com outras pessoas e afeto negativo e positivo. Concluiu-se a importância da interação social e do número de pessoas que o idoso convive para uma percepção do seu bem-estar subjetivo e para sua capacidade funcional.

palavras-chave

Apoio social. Qualidade de vida. Funcionalidade. Idoso.

\section{Introdução}

O crescimento populacional de idosos é um fenômeno atual nos países em desenvolvimento, onde se observa um envelhecimento acelerado da população nas últimas décadas acompanhado da diminuição das taxas de natalidade e aumento da expectativa de vida. Neste contexto, observa-se que o envelhecimento deixa de ser uma situação vivida pelos países desenvolvidos, como os países norte-americanos e europeus, e passa a ser também uma situação característica de países em desenvolvimento. No Brasil, os idosos representam aproximadamente $11 \%$ da população geral. O Censo de 2010 relatou que, dos 190 milhões de brasileiros, mais de 20,6 milhões têm 60 anos ou mais (IBGE, 2010), e projeções apontam um crescimento desse grupo populacional para 25 milhões até 2025 (GARRIDO; MENEZES, 2002; VERAS, 2009).

De maneira geral, o envelhecimento se caracteriza como um processo dinâmico, progressivo e irreversível, no qual estão envolvidos fatores biológicos como alterações celulares e genéticas; fatores e contextos ambientais compostos principalmente pelo estilo de vida; e fatores psicossociais que englobam as relações sociais, os papéis desenvolvidos na comunidade e o estado emocional do idoso (LITVOC; BRITO, 2004). Com o processo de envelhecimento, há mudanças nas condições de saúde da população como aumento de doenças crônico-degenerativas, morbidade, incapacidade funcional e mortalidade. Estas mudanças possuem um grande impacto sobre as famílias, as formas de cuidado e sobre o sistema de saúde (PARAHYBA; SIMÕES, 2006). 
Estudos realizados na população brasileira mostram que há aumento do nível de incapacidade funcional e diminuição da qualidade de vida entre os idosos (MELZER; PARAHYBA, 2004; LEBRÃO; LAURENTI, 2005; PARAHYBA; SIMÕES, 2006; CARNEIRO et al., 2007; CHAIMOWICZ, 2007). Lebrão e Laurenti (2005) demonstraram que mais de $20 \%$ dos idosos da amostra de seu estudo apresentavam limitações funcionais que impediam o autocuidado e o desempenho de atividades de vida diária (AVD) e atividades instrumentais de vida diária (AIVD), necessitando de ajuda de terceiros. O comprometimento da capacidade funcional do idoso tem implicações importantes para a família, comunidade, para o sistema de saúde e para a vida do próprio idoso, uma vez que a incapacidade ocasiona maior vulnerabilidade e dependência na velhice, contribuindo para a diminuição do bem-estar e da qualidade de vida dos idosos.

Em um estudo, Albuquerque e Tróccoli (2004) identificaram que a qualidade de vida do idoso pode influenciar diversos fatores como condição de saúde, capacidade funcional, estado emocional, assim como pode sofrer influências de interações sociais, atividade intelectual e bem-estar físico e mental. Carneiro et al. (2007) apontam que a qualidade de vida está associada com diversos componentes, como o estado emocional, cognitivo, interação social e autoproteção de saúde. Tais aspectos são indispensáveis para a realização de atividades básicas e instrumentais de vida diária, integrando a relação entre funcionalidade e bem-estar subjetivo (ALMEIDA, 2010).

Atualmente, investigações na área da saúde do idoso têm apontado para a importância da relação entre funcionalidade, qualidade de vida e bem-estar. Rosa et al. (2007) mostrou que uma melhor qualidade de vida do idoso está relacionada à manutenção da capacidade funcional e à uma rede de interação social estruturada. Além disso, a literatura tem apontado uma relação entre menor rede de apoio social e pior funcionalidade, bem como de menor rede de apoio social com pior bem-estar em idosos da comunidade (CARNEIRO et al., 2007; ALVARENGA et al., 2011). De forma geral, rede social diz respeito ao grupo de pessoas que o indivíduo mantém contato ou vínculo social; já o apoio social refere-se a pessoas na sua rede com quem o indivíduo possa contar em situações mais difíceis, recebendo algum tipo de auxílio, seja material, emocional ou afetivo (GRIEP, 2003). Este é um processo recíproco que gera efeitos positivos tanto para o sujeito que recebe como também para quem oferece o apoio, permitindo que ambos tenham mais sentido de controle sobre suas vidas (PEDRO; ROCHA; NASCIMENTO, 2008; RESENDE et al., 2006). Para além, dar e oferecer este apoio está associado à capacidade do indivíduo em adaptar-se às perdas ligadas ao processo de envelhecimento e em recuperar-se de eventos estressantes ao longo do curso de vida (FREIRE, 2001). Observa-se, 
assim, que as redes de apoio social são fortemente associadas com resultados positivos e estão relacionadas com saúde, adaptação psicológica, percepção de bem-estar, redução do mal-estar, longevidade e mortalidade, satisfação com a vida, entre outros (RAMOS, 2002; SILVA et al., 2003).

Esses dados sustentam a importância do apoio social para o bem-estar e para a manutenção da funcionalidade na velhice. Assim, com o envelhecimento populacional, o aumento da expectativa de vida e do número de doenças crônico-degenerativa na população de idosos, as investigações sobre envelhecimento saudável, bem-estar subjetivo, funcionalidade e apoio social na velhice tornaram-se focos da atenção de diversos pesquisadores e profissionais da saúde. Neste contexto, o objetivo deste estudo é avaliar o impacto do apoio social na funcionalidade e no bem-estar subjetivo de idosos na comunidade.

\section{Materiais e método}

Este foi um estudo observacional do tipo transversal que buscou investigar as relações entre rede de apoio social, bem-estar subjetivo e funcionalidade entre idosos da comunidade residentes do município de Uberaba (MG). Uberaba apresenta uma localização estratégica do ponto de vista geoeconômico, é considerada polo de desenvolvimento agropecuário e industrial e registra uma população de 288.235 habitantes. Segundo o Censo/IBGE, o município de Uberaba apresentava, em 2010, uma população idosa de 29.855 pessoas, correspondendo a aproximadamente $10,4 \%$ da população (IBGE, 2010).

Foram selecionados para participar deste estudo, idosos residentes em Uberaba (MG), cadastrados nas equipes de Estratégia Saúde da Família de duas Unidades de Saúde da Família (USF) e idosos participantes de diversas comunidades e associações, quais foram entrevistados em seus domicílios. As USFs são a porta de entrada para o sistema público de saúde e fornecem à população ações de saúde focadas na atenção básica. Já as comunidades e associações de moradores são consideradas redes de apoio à saúde, suporte político, religioso ou social e meio de manutenção de qualidade de vida. As duas USFs e a associação participantes deste estudo estão localizadas em regiões sociodemográficas pertencentes aos três distritos sanitários de abrangência da Secretaria Municipal de Saúde de Uberaba/MG (SMS-Uberaba).

Os critérios de inclusão para a realização deste estudo foram: pessoas de ambos os sexos, com idade igual ou superior a 60 anos, que concordassem em participar e assinar o termo de consentimento livre e esclarecido aprovado pelo Comitê de Ética em Pesquisa da Universidade Federal do Triângulo 
Mineiro (UFTM) (parecer no 2459) e pela Secretaria de Saúde de Uberaba. Neste estudo, os idosos que estavam frequentando a USF ou envolvidos em alguma atividade na associação eram convidados para participar da pesquisa, sendo excluídos aqueles que não se encaixavam nos critérios de inclusão desta, ou não dispunham de tempo para responder aos questionários, compondo, assim, uma amostra por conveniência.

As variáveis demográficas e socioeconômicas dos idosos foram identificadas através de um questionário desenvolvido especificamente para este estudo, o qualis englobava informações sobre idade; sexo; escolaridade; estado civil; arranjo familiar; número de filhos vivos; benefícios recebidos como aposentadoria, pensão, benefício de prestação continuada, entre outros; renda da família e número de pessoas que residem no domicílio.

Para mensuração do bem-estar foi utilizada a Escala de Bem-Estar Subjetivo (EBES), validada para o contexto brasileiro por Albuquerque e Trócolli (2004). Esta escala é composta por 69 itens que buscam mensurar dois componentes do bem-estar subjetivo, a saber: satisfação com a vida e afetos positivos e afetos negativos. Os componentes afetos positivos e afetos negativos, utilizados neste estudo, informam a frequência de reações emocionais afetivas positivas e negativas que o entrevistado tem com sua rede de apoio (ALBUQUERQUE; TRÓCOLLI, 2004). Esta subescala abrange 47 itens para a mensuração dos afetos positivos e negativos, sendo que o escore final para cada dimensão é calculado a partir da soma das respostas daquele fator, dividido pelo número de itens correspondente do mesmo (FERNANDES; RAPOSO; FERNANDES, 2012). Para a análise dos resultados da subescala do afeto positivo, quanto maior a média do escore obtida pelo indivíduo, maior será seu bem-estar em relação à vida. Já para a análise do afeto negativo, quanto maior a pontuação obtida, pior será o bem-estar percebido.

O apoio social dos idosos foi mensurado a partir de duas medidas: uma qualitativa e outra quantitativa. A medida qualitativa avaliou a percepção do apoio social utilizando um domínio do instrumento Perfil de Saúde de Nottingham (PSN), o qual avalia a Interação Social do entrevistado, sendo que este domínio do instrumento consiste em cinco questões. Originalmente, o PSN foi desenvolvido para avaliar qualidade de vida em pacientes com doenças crônicas (BRITTO; SANTOS; BUENO, 2002). Este instrumento consiste em um questionário composto por 38 itens, baseados na Classificação Internacional de Funcionalidade, Incapacidade e Saúde (CIF) descrita pela Organização Mundial de Saúde (OMS) (OMS, 2003). As perguntas desta escala se apresentam no formato sim/não e seus itens avaliam seis categorias relacionadas com a qualidade de vida, sendo estas: nível de energia e de fadiga (NE); dor, sua intensidade 
e interferência nas AVDs (D); reações emocionais que englobam questões sobre ansiedade, depressão, alterações no comportamento ou descontrole emocional e bem-estar psicológico (RE); sono, sua qualidade e a presença de insônia (S); habilidades físicas e a presença de limitações na realização das AVDs (HF); e interação social (IS), que avalia a existência do sentimento de solidão e a dificuldade de interagir com outras pessoas (TEIXEIRA-SALMELA et al., 2004). Já a medida quantitativa do apoio social dos entrevistados foi avaliada pelo número de pessoas que coabitavam na mesma residência que o idoso.

A avaliação funcional dos idosos foi realizada pelo Brazilian Functional Assessment Questionnarie (BOMFAQ), a versão brasileira do questionário Older Americans Resources and Services Program (OARS) (PITON, 2004). Este instrumento tem por finalidade quantificar as dificuldades que o idoso apresenta em realizar suas AVDs e AIVDs, tais como deitar e levantar-se da cama, comer, pentear o cabelo, andar no plano, tomar banho, vestir-se, ir ao banheiro em tempo, subir um lance de escada, fazer compras, preparar refeições, sair de condução, entre outros. O BOMFAQ avalia, assim, o grau de autonomia do idoso e indica se este é independente ou apresenta dependência leve, moderada ou grave (PITON, 2004; SANTOS, 2011).

Em relação à análise estatística, inicialmente as variáveis investigadas foram descritas para que houvesse a caracterização da amostra, sendo para isso utilizadas medidas descritivas (média, desvio padrão, mínimo e máximo) para as variáveis quantitativas e, para as variáveis categóricas, foram utilizadas medidas de frequência absoluta e frequência relativa. Para avaliar associação entre Interação Social (IS), Capacidade Funcional (CF) e Bem-Estar Subjetivo (BES) foi utilizado o coeficiente de correlação de Pearson. Para todos os testes estatísticos, foi utilizado o valor de significância igual a 5\%. As análises foram realizadas no software estatístico SPSS (Statistical Package for Social Sciences) versão 20.0.

\section{Resultados}

A coleta de dados foi realizada no período de abril a junho de 2013, sendo a amostra composta por 39 idosos, com idade variando de 60 a 82 anos, atingindo uma média de idade amostral igual a 68 anos $(\mathrm{DP}=6,32)$. Destes idosos $29(74,4 \%)$ eram do sexo feminino e 10 (25,6\%) do sexo masculino, sendo que $12(30,8 \%)$ eram casados e $16(41,0 \%)$ eram viúvos. Em relação à escolaridade dos participantes, quatro (10,3\%) não haviam estudado, 16 (41\%) tinham ensino fundamental incompleto, enquanto $12(30,8 \%)$ possuíam ensino fundamental completo. Um (2,6\%) tinha ensino médio incompleto e seis $(15,4 \%)$ terminaram o ensino médio. Nenhum dos participantes entrevistados relatou possuir 
ensino superior. A maioria dos idosos entrevistados nessa pesquisa estava aposentada $30(76,9 \%)$, e entre esses, $16(41,0 \%)$ realizavam alguma atividade de trabalho remunerada. A renda mensal da amostra oscilou de zero a 5.500,00, com média igual a $1.179,70$ reais por mês $(\mathrm{DP}=1.011,77)$, valor que corresponde a 1,73 salários mínimos mensais. Quanto aos hábitos de vida, 24 idosos eram sedentários (61,5\%), cinco eram tabagistas (12,8\%) e seis faziam ingestão regular de bebidas alcoólicas (15,4\%). A tabela 1 apresenta a descrição completa das características sócio-demográficas, ocupacionais e de hábitos de vida dos participantes deste estudo.

Tabela 1 - Características Sócio demográficas, Ocupacionais e de Hábitos de Vida dos Idosos ( $n=39)$. Uberaba, 2013.

\begin{tabular}{|c|c|c|c|}
\hline Variáveis & Categorias & Frequência & Porcentagem \\
\hline \multirow{2}{*}{ Sexo } & Feminino & 29 & 74,4 \\
\hline & Masculino & 10 & 25,6 \\
\hline \multirow{4}{*}{ Estado Civil } & Solteiro & 4 & 10,3 \\
\hline & Casado & 12 & 30,8 \\
\hline & Separado/Divorciado & 7 & 17,9 \\
\hline & Viúvo & 16 & 41,0 \\
\hline \multirow{4}{*}{ Com quem mora } & Sozinho & 18 & 46,2 \\
\hline & Cônjuge & 10 & 25,6 \\
\hline & Outros familiares & 9 & 23,1 \\
\hline & Cônjuge e familiares & 2 & 5,1 \\
\hline \multirow{5}{*}{ Escolaridade } & Não estudou & 4 & 10,3 \\
\hline & Ensino fundamental incompleto & 16 & 41,0 \\
\hline & Ensino fundamental completo & 12 & 30,8 \\
\hline & Ensino médio incompleto & 1 & 2,6 \\
\hline & Ensino médio completo & 6 & 15,4 \\
\hline \multirow{2}{*}{ Aposentadoria } & Não & 9 & 23,1 \\
\hline & Sim & 30 & 76,9 \\
\hline \multirow{2}{*}{ Trabalho } & Não & 23 & 59,0 \\
\hline & Sim & 16 & 41,0 \\
\hline \multirow{2}{*}{ Atividade física regular } & Não & 24 & 61,5 \\
\hline & Sim & 15 & 38,5 \\
\hline \multirow{2}{*}{ Fumantes } & Não & 34 & 87,2 \\
\hline & Sim & 5 & 12,8 \\
\hline \multirow{2}{*}{ Consome bebida alcoólica } & Não & 33 & 84,6 \\
\hline & Sim & 6 & 15,4 \\
\hline
\end{tabular}


A análise quantitativa do apoio social foi calculada pelo número de pessoas que residiam na mesma casa que o idoso. Este número variou de 0 a 4 pessoas por casa, com média de 1,69\% (DP =1,36), sendo que dezoito $(46,2 \%)$ dos idosos moravam sozinhos. Já para análise qualitativa do apoio social percebido pelos idosos foi utilizado o domínio de IS do PSN, o qual apresentou uma variação de 0 a 60 pontos, com média de 18,97\% ( $\mathrm{DP}=23,37)$. Considerando que quanto maior o escore do domínio de IS do PSN, pior o apoio social percebido, este resultado aponta que a amostra percebe sua interação social como moderada.

A capacidade funcional avaliada através do BOMFAQ teve uma variação de 0 a 18 pontos, com média de 4,38 ( $\mathrm{DP}=3,51)$. Por fim, na análise descritiva do afeto positivo houve variação de 2,09 a 3,86 pontos, com média de 2,92 $(\mathrm{DP}=0,43)$ e, para o afeto negativo, a variação foi de 2,16 a 3,80 pontos, com média de $2,90(\mathrm{DP}=0,40)$.

A funcionalidade apresentou correlação estatisticamente significativa, positiva e de intensidade baixa com o escore de IS ( $p=0,025, r=0,359)$, indicando que uma boa percepção de interação social está associada com uma melhor capacidade funcional dos participantes (Figura 1). Não houve associação significativa entre o número de pessoas coabitantes na mesma casa e o escore final do BOMFAQ. 
Figura 1 - Associação entre Interação Social e Capacidade Funcional de Idosos Residentes na Comunidade $(n=39)$. Uberaba, 2013

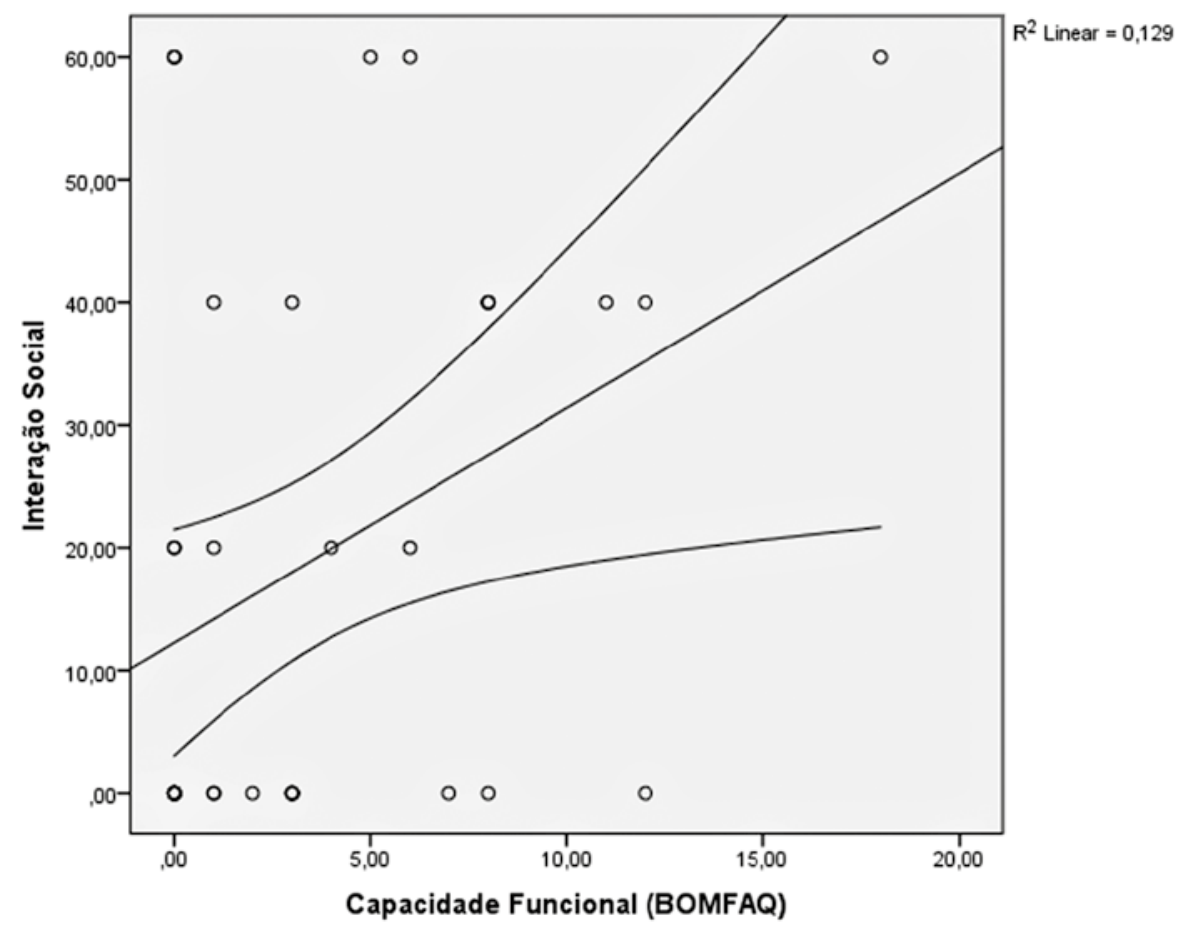


O bem-estar subjetivo foi avaliado por dois domínios da Escala de Bem-Estar Subjetivo (EBES): afeto positivo e afeto negativo. Nota-se uma correlação importante encontrada no estudo entre o número de pessoas que moram com o idoso entrevistado e a percepção do afeto positivo em situações cotidianas $(\mathrm{p}=0,000$ e $\mathrm{r}=0,553)$, apontando que quanto maior o número de pessoas que coabitavam na mesma residência, maior é a percepção de afeto positivo recebido pelo idoso (Figura 2). Igualmente, foi encontrada uma correlação significativa entre o número de pessoas que moram na mesma casa e a percepção do afeto negativo ( $p=0,000$ e $r=0,550)$, indicando que quanto maior o número de pessoas que coabitam a mesma residência pior é a percepção de afeto negativo recebido pelo idoso (Figura 3). Nesta amostra não houve significância estatística entre afeto positivo e afeto negativo $(p=0,254$ e $r=0,187 ; p=0,203$ e $r=0,208$, respectivamente) quando associadas ao apoio social percebido pelos idosos.

Figura 2 - Associação entre Número de Pessoas que Residem na mesma Casa e Sentimento de Afeto Positivo de Idosos Residentes na Comunidade ( $n=39)$. Uberaba, 2013.

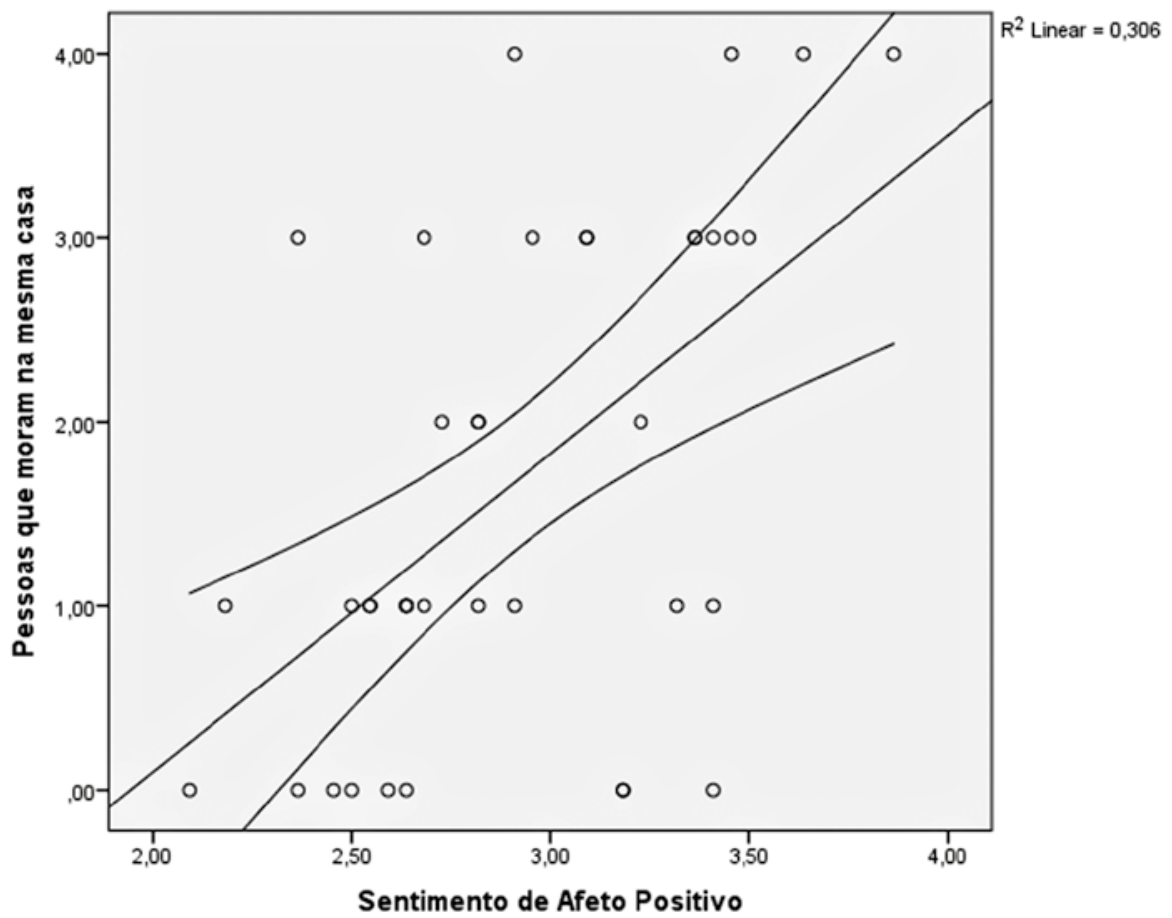


Figura 3 - Associação entre Número de Pessoas que Residem na mesma Casa e Sentimento de Afeto Negativo de Idosos Residentes na Comunidade ( $n=39)$. Uberaba, 2013.

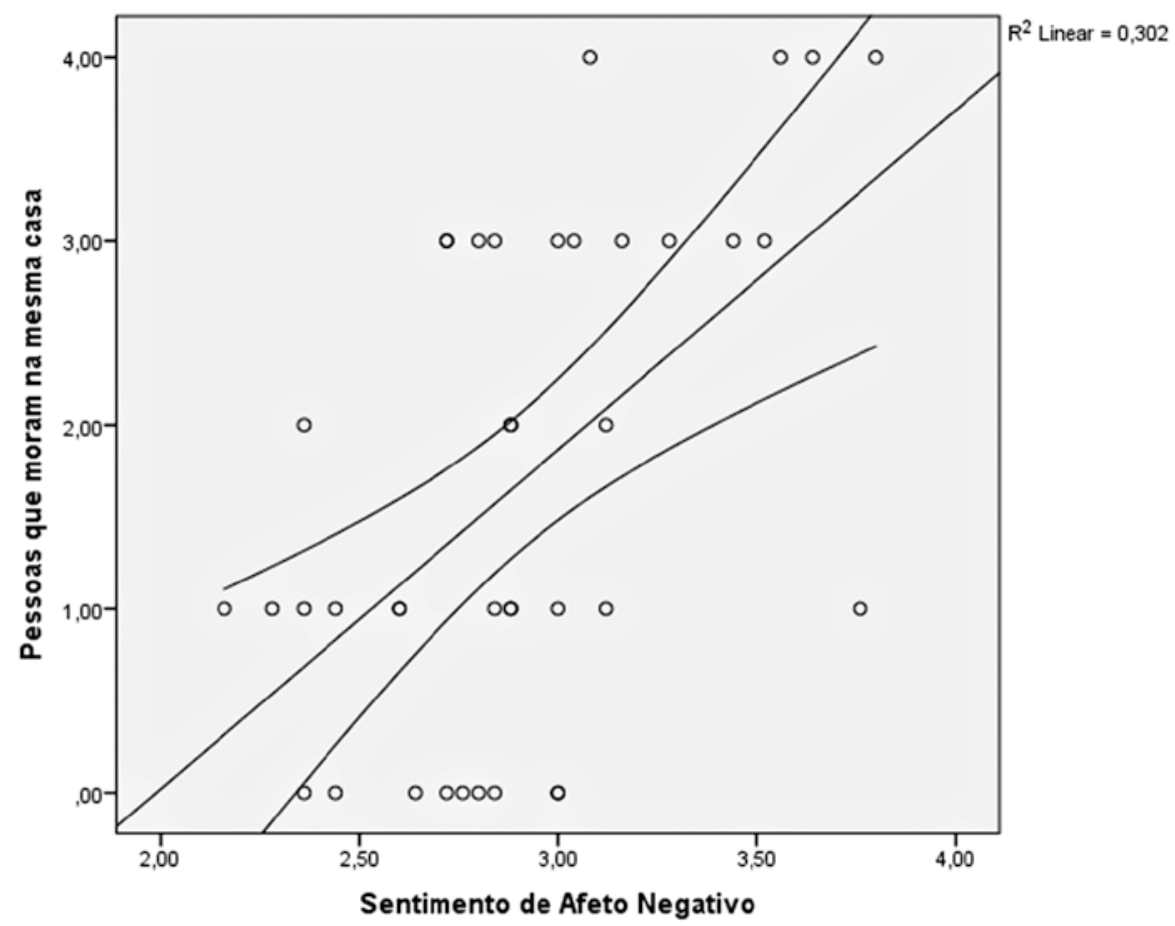

\section{Discussão}

No presente estudo, observou-se a predominância da população feminina, o que está associado ao perfil de envelhecimento demográfico brasileiro interligado à questão de gênero. Essa prevalência é indicada como consequência do índice de mortalidade masculina ser maior que a mortalidade feminina, ou seja, os idosos homens possuem uma expectativa de vida menor do que as mulheres (SERASA, 2008). Os padrões de procura e de uso dos serviços de saúde entre os idosos brasileiros também são determinados por outros fatores que afetam diferenciadamente homens e mulheres. Há evidência de que as mulheres idosas suportam uma maior carga de condições crônicas de doença, 
o que acarreta um declínio funcional mais acentuado (PARAHYBA; VERAS, 2008). Além dos fatores comportamentais, que indicam uma maior procura das mulheres por serviços de saúde do que os homens, mesmo quando controlado o efeito da restrição de atividades por motivo de saúde (PARAHYBA, 2006; TRAVASSOS et al., 2002).

A média de renda dos idosos encontrada nessa amostra $(\mathrm{R} \$ 1.179,70)$ apresentou ser maior do que a média apontada pelo IBGE (2010) (R\$ 657,00). Esses dados apontam que esta população ocupa lugar de destaque no modelo de organização financeira e orçamentária da família brasileira. Através de uma comparação realizada entre os Censos, nos anos de 2002 e 2010, foi identificado que $62,4 \%$ dos idosos eram responsáveis financeiramente pelo seu domicílio. Apesar da renda apresentada pela amostra, quase metade dos idosos entrevistados estão inseridos em atividades laborais, esta participação não acontece apenas devido à influência que o idoso pode ter no mercado de trabalho, mas, sobretudo como um fator do seu processo de independência. O retorno da população idosa para o mercado de trabalho é uma realidade brasileira se comparada com outros países, principalmente os desenvolvidos. Segundo Camarano (2001) e Carrera-Fernandez e Menezes (2001), isto ocorre devido ao aumento de renda familiar e o papel desempenhado pelo idoso a fim de permanecer como mantenedor e líder financeiro de seu domicílio. $\mathrm{O}$ elevado número de unidades domésticas unipessoais, presentes nesta amostra $(23,1 \%)$ foi algo de destaque no estudo, uma vez que em países em desenvolvimento, como o Brasil, a porcentagem de idosos que vivem com seus filhos ou outros familiares continua sendo elevada, processo inverso que acontece nos países desenvolvidos onde a proporção de idosos vivendo com seus familiares é menor (UNITED NATIONS, 2005). Via de regra, o que se pode observar é que, quanto maior o nível de desenvolvimento econômico do país ou quanto maior a renda do idoso, menores são as taxas de corresidência entre os idosos (UNITED NATIONS, 2005; CAMARGOS; MACHADO; RODRIGUES, 2007). Para Camarano e El Ghaouri (2003) e Camargos, Machado e Rodrigues (2007), a tendência de redução do número de filhos, aumento de divórcios, mudanças de estilo de vida, melhora nas condições de saúde dos idosos e consequente ampliação da longevidade e, principalmente, a viuvez são fatores explicativos de parte do crescimento das famílias unipessoais

Outro achado importante deste estudo foi a associação significativa entre percepção do apoio social recebido com um maior nível de independência e funcionalidade do idoso, ou seja, quanto mais fortalecida era a percepção do idoso sobre a rede de apoio social maior era a sua capacidade funcional. Manter a capacidade funcional é uma das referências para um envelhecimento 
bem-sucedido e para uma boa qualidade de vida dessa população, sendo que a perda funcional está associada com uma maior fragilidade e dependência na realização de suas AVD e AIVD (GUIMARÃES et. al., 2004). Segundo Canesqui e Barsaglini (2012), a interação social pode contribuir para a promoção e manutenção da saúde física e mental do idoso, favorecer adesão a novos tratamentos, melhorar a qualidade de vida e criação de novas estratégias para lidar com doenças crônicas. Além disto, a manutenção de relacionamentos estáveis com amigos e confidentes diminuem o risco da perda funcional nesta população (D'ORSI; XAVIER; RAMOS, 2011). Neste contexto, o sentimento de solidão pelo idoso e dificuldades de interagir com outras pessoas estão associados a uma perda significativa da capacidade funcional, indicando a importância do sentimento de apoio e interação social para sua funcionalidade.

A relação entre o número de pessoas que residem no mesmo domicílio e a percepção dos idosos em relação e ao seu bem-estar apresentou duas vertentes distintas. Os resultados sugerem que conviver com mais familiares está associado significativamente a um maior afeto positivo por oferecer suporte, assistência e proteção, proporcionados pela família, que pode ser considerada como um sistema no qual é possível identificar interação e contribuições entre seus membros (SILVA; GALERA; MORENO, 2007). Ao mesmo tempo, os resultados apontam que este convívio contínuo com seus familiares também se associa a uma percepção negativa de bem-estar. Segundo Scorsolini-Comin, Santos e Souza (2012) isso ocorre devido a capacidade que o idoso possui em distinguir momentos transitórios de prazer, felicidade e momentos de ansiedade, aborrecimento e pessimismo. O julgamento da existência desses diferentes sentimentos e percepções podem acontecer quando o objetivo do sistema familiar não é alcançado e a convivência com diversas pessoas pode levar a situações conflituosas existentes no contato diário. Em atenção a essas percepções distintas de afeto positivo e negativo, observa-se a importância do papel dessa rede social na condição de saúde e qualidade de vida do idoso, já que este apoio é capaz de satisfazer suas necessidades sociais, além de possibilitar o engajamento social dessa população (NERI, 2005).

\section{5 conclusão}

Observou-se no estudo que a interação social possui aspectos qualitativos e quantitativos que influenciam na funcionalidade e bem-estar dos idosos. A quantidade de pessoas com que o idoso convive interfere com resultados significativos em seu bem-estar subjetivo. Já a percepção de apoio e suporte 
social pelo idoso se mostrou associada a melhor funcionalidade e mais independência. Assim, além da qualidade do suporte percebido pelo idoso, devemos ressaltar a relevância de fortalecer e ampliar o apoio social para que ocorra uma melhoria na capacidade funcional desta população.

Foi significativa a associação da capacidade funcional quando a rede de suporte do idoso era mais fortalecida, atentando para a necessidade do desenvolvimento de estratégias para fortalecimento da rede de suporte social do idoso, uma vez que a interação social se mostra como um fator de proteção da perda da capacidade funcional.

Por fim, os resultados deste estudo apontam para percepções tanto positivas quanto negativas de bem-estar quando se tem mais de um morador coabitando na mesma casa. Ainda são escassos os estudos que investigam o impacto de moradias multigeracionais no cotidiano do idoso, em especial, em sua funcionalidade e bem-estar. Sugere-se que futuras investigações apontem quais são os mecanismos e efeitos dessa coabitação e os possíveis fatores mediadores da relação entre rede de apoio social e capacidade funcional, bem-estar e condição de saúde geral na população de idosos.

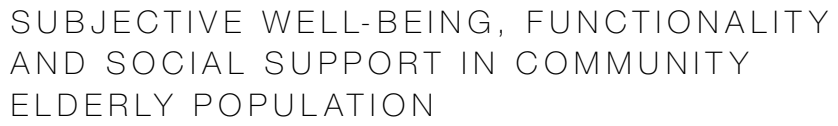

abstract

The Aging process is one in which can be recognized for some of its characteristics such as functional disability increase and poorer quality of life. This process can be influenced by the Social Network Support and by the well-being of the elderly. This present study aim was to evaluate the social support impact on functionality and subjective well-being of the elderly in the society. Thus a quantitative, observational and cross-sectional study with 29 seniors enrolled in strategy teams of family and health support and also members of the Community Association in Uberaba (MG), was done. It was assessed Social Support by the Social Interaction domain of the Nottingham Health Profile, functionality using the BOMFAQ and welfare through the Subjective well-being scale. The sample mainly featured women (74.5\%), between the ages of 60 and 82, married or living with their partners, with no or very little schooling and average monthly income of $\mathrm{R} \$ 1.179,90$. The analysis has shown a strong correlation between Interaction and Social Support and Functional capability as well as 
a considerable correlation between living with others and positive/ negative affection. With this study it has been found the significance of the Social Interaction and the number of people to whom the elderly live with for a perception of their subjective well-being and functional capability.

keywords

Social Support. Quality of Life. Functionality. Elderly.

\author{
referências
}

ALBUQUERQUE, Anelise Salazar; TRÓCCOLI, Bartholomeu Tôrres. Desenvolvimento de uma Escala de Bem-Estar Subjetivo. Psicologia: Teoria e Pesquisa, Brasília, v. 20, n. 2, p. 153-164, maio/ago. 2004.

ALMEIDA, Ana Rita Soares de. Práticas desportivas, bem-estar subjetivo e autopercepções físicas em idosos. 2010. 148 f. Dissertação (Mestrado em Atividade Física para a Terceira Idade) - Faculdade de Desporto, Universidade do Porto, Porto, 2010

ALVARENGA, Márcia Regina Martins et al. Rede de suporte social do idoso atendido por equipes de Saúde da Família. Ciência \& Saúde Coletiva, Rio de Janeiro, v. 16, n. 5, p. 2603-2611, maio 2011.

BRITTO, Raquel Rodrigues; SANTOS, Cynthia Fernandes Ferreira; BUENO, Flavia Faria. Reabilitação pulmonar e qualidade de vida dos pacientes portadores de DPOC. Revista Brasileira de Fisioterapia da Universidade de São Paulo, São Paulo, v. 9, p. 9-16, jan./jun. 2002.

CAMARANO, Ana Amélia. O idoso brasileiro no mercado de trabalho. Rio de Janeiro: IPEA, 2001.

CAMARANO, Ana Amélia; EL GHAOURI, Solange Kanso. Famílias com idosos: ninhos vazios? Rio de Janeiro: IPEA, 2003.

CAMARGOS, Mirela Castro Santos; MACHADO, Carla Jorge; RODRIGUES, Roberto do Nascimento. A relação entre renda e morar sozinho para idosos paulistanos 2000. Revista Brasileira de Estudos de População, São Paulo, v. 24, n. 1, p. 37-51, jan./jun. 2007.

CANESQUI, Ana Maria; BARSAGLINI, Reni Aparecida. Apoio social e saúde: pontos de vista das ciências sociais e humanas. Ciência \& Saúde Coletiva, Rio de Janeiro, v. 17, n. 5, p. 1103-1114, maio 2012.

CARNEIRO, Rachel Shimba et al. Qualidade de vida, apoio social e depressão em idosos: relação com habilidades sociais. Psicologia: reflexão e crítica, Porto Alegre, v. 20, n. 2, p. 229-237, maio/ago. 2007

CARRERA-FERNANDEZ, José; MENEZES, Wilson F. O idoso no mercado de trabalho: uma análise a partir da região metropolitana de Salvador. Revista Econômica do Nordeste, Fortaleza, v. 32, n. 1, p. 52-67, jan./mar. 2001.

CHAIMOWICZ, Flávio. A saúde dos idosos brasileiros às vésperas do século XXI: problemas, projeções e alternativas. Revista de Saúde Pública, São Paulo, v. 31 n. 2, p. 184-200, abr. 1997. 
D'ORSI, Eleonora; XAVIER, André Junqueira; RAMOS, Luiz Roberto. Trabalho, suporte social e lazer protegem idosos da perda funcional: Estudo Epidoso. Revista Saúde Pública, São Paulo, v. 45, n. 4, p. 685-92, ago. 2011

FERNANDES, Marcos Gimenes; VASCONCELOS-RAPOSO, José; FERNANDES, Helder Miguel. Relação entre orientações motivacionais, ansiedade e autoconfiança, e bem-estar subjetivo em atletas brasileiros. Motricidade, Vila Real, v. 8, n. 3, p. 4-18, jul. 2012.

FREIRE, Sueli Aparecida. Bem estar subjetivo e metas de vida: um estudo transversal com homens e mulheres pertencentes a três faixas de idade. 2001. $167 \mathrm{f}$. Tese (Doutorado em Educação) - Faculdade de Educação, Universidade Federal de Campinas, Campinas, 2001

GARRIDO, Regiane; MENEZES, Paulo R. O Brasil está envelhecendo: boas e más notícias por uma perspectiva epidemiológica. Revista Brasileira de Psiquiatria, São Paulo, v. 24, supl.1, p. 3-6, abr. 2002.

GRIEP, Rosane Harter. Confiabilidade e Validade de Instrumentos de Medida de Rede Social e de Apoio Social Utilizados no Estudo Pró-Saúde. 2003. 177 f. Tese (Doutorado em Ciências na área de Saúde Pública) - Escola Nacional de Saúde Pública, Fundação Oswaldo Cruz, Rio de Janeiro, 2003.

GUIMARÃES, Laiz Helena de Castro Toledo et al. Avaliação da capacidade funcional de idosos em tratamento fisioterapêutico. Revista Neurociências, São Paulo, v. 12, n. 3, jul./set. 2004.

INSTITUTO BRASILEIRO DE GEOGRAFIA E ESTATÍSTICA. Censo Demográfico 2010. Rio de Janeiro: IBGE, 2010. Disponível em: <http://www. bge.gov.br/home/>. Acesso em: 11 jul. 2013.

LEBRÃO, Maria Lúcia; LAURENTI, Rui. Saúde, bem-estar e envelhecimento: o estudo SABE no município de São Paulo. Revista Brasileira de Epidemiologia, São Paulo, v. 8, n. 2, p. 127-141, jun. 2005.

LITVOC, Júlio; BRITO, Francisco Carlos de. Envelhecimento: Prevenção e Promoção da Saúde. São Paulo: Editora Atheneu, 2004.

MELZER, David; PARAHYBA, Maria Isabel. Socio-demographic correlates of mobility disability in older Brazilians: results of the first national survey. Age and Ageing, Oxford, v. 33, n. 3, p. 253-259, Mar. 2004

NERI, Anita Liberalesso. Palavras-chave em gerontologia. 5. ed. Campinas: Alínea, 2005.

ORGANIZAÇÃO MUNDIAL DE SAÚDE. CIF: Classificação Internacional de Funcionalidade, Incapacidade e Saúde. São Paulo: EDUSP, 2003.

PARAHYBA, Maria Isabel. Desigualdades de gênero em saúde entre os idosos no Brasil. In: ENCONTRO NACIONAL DE ESTUDOS POPULACIONAIS, 15., 2006, Caxambu. Anais... Minas Gerais: ABEP, 2006. p. 1-11.

PARAHYBA, Maria Isabel; SIMÕES, Celso Cardoso da Silva. A prevalência de incapacidade funcional em idosos no Brasil. Ciência e Saúde coletiva, Rio de Janeiro, v. 11, n. 4, p. 967-974, dez. 2006.

PARAHYBA, Maria Isabel; VERAS, Renato. Diferenciais sociodemográficos no declínio funcional em mobilidade física entre os idosos no Brasil. Ciência e Saúde coletiva, v. 13, n. 4, p. 1257-1264, ago., 2008 ,

PEDRO, lara Cristina da Silva; ROCHA, Semiramis Melani Melo: NASCIMENTO, Lucila Castanheira. Apoio e rede social em enfermagem familiar. Revista Latino-americana de Enfermagem, Ribeirão Preto, v. 16, n. 2, p. 324-7, abr. 2008 
PITON, Danielle Alexandra. Análise dos Fatores de Risco de Quedas em Idosos: estudo exploratório em instituição de longa permanência no município de Campinas. 2004. 103 f. Dissertação (Mestrado em Gerontologia) - Faculdade de Educação, Universidade Estadual de Campinas, Campinas, 2004.

RAMOS, Marília P. Apoio social e saúde entre idosos. Sociologias, Porto Alegre, v. 4, n. 7, p. 156-175, jan./jun. 2002.

RESENDE, Marineia Crosara de et al. Rede de relações sociais e satisfação com a vida de adultos e idosos. Psicología para América Latina, São Paulo, v. 5, n. 5, supl.1, p. 1-20, fev. 2006.

ROSA, Tereza Etsuko da Costa et al. Aspectos estruturais e funcionais do apoio social de idosos do Município de São Paulo, Brasil. Cadernos de Saúde Pública, Rio de Janeiro, v. 23, n. 12, p. 2982-2992, dez. 2007.

SANTOS, Renata Newman Leite Cardoso dos. Análise da capacidade funcional e do estado cognitivo em idosos do município de Campina Grande - PB. 2011. 34 f. Trabalho de Conclusão de Curso (Monografia) - Centro de Ciências Biológicas e da Saúde, Curso de Graduação em Fisioterapia, Universidade Estadual da Paraíba, João Pessoa, 2011.

SCORSOLINI-COMIN, Fabio; SANTOS, Manoel Antônio dos; SOUZA, Roberto Molina de. Expressão do afeto e bem-estar subjetivo em pessoas casadas. Estudos de Psicologia, Natal, v. 17, n. 2, p. 321-328, maio/ago. 2012.

SERASA. Guia Serasa de orientação ao cidadão. São Paulo: Serasa, 2013. Disponível em: <http://www.serasaexperian.com.br/guiaidoso/>. Acesso em: 09 ago. 2013.

SILVA, Isabel et al. Efeitos do apoio social na qualidade de vida, controle metabólico e desenvolvimento de complicações crônicas em indivíduos com diabetes. Psicologia, Saúde e Doenças, Lisboa, v. 4, n. 1, p. 21-32, jul. 2003.

SILVA, Lucía; GALERA, Sueli Aparecida Frari; MORENO, Vânia. Encontrando-se em casa: Uma proposta de atendimento domiciliar para famílias de idosos dependentes. Acta Paulista de Enfermmagem, São Paulo, v. 20, n. 4, p. 397-403, dez. 2007.

TEIXEIRA-SALMELA, Luci Fuscaldi et al. Adaptação do Perfil de Saúde de Nottingham: um instrumento simples de avaliação da qualidade de vida. Cadernos de Saúde Pública, Rio de Janeiro, v. 20, n. 4, p. 905-914, ago. 2004

TRAVASSOS, Cláudia; VIACAVA, Francisco; PINHEIRO, Rejane; BRITO, Alexandre. Utilização dos serviços de saúde no Brasil: gênero, características familiares e condição social. Revista Panamericana de Saúde Publica, Washington, v. 11, n. 5/6, p. 365-373, jun. 2002.

UNITED NATIONS. Living arrangements of older persons around the world. New York: United Nations, 2005.

VERAS, Renato. Envelhecimento populacional contemporâneo: demandas, desafios e inovações. Revista Saúde Pública, São Paulo, v. 43, n. 3, p. 548-554, maio/jun. 2009.

Recebido em: 01/11/2013

Aceite final: 26/11/2014 